nutrients ( 0.5 to $100 \mathrm{~mL}$; daily performance qualification) over 3 consecutive days. The concentration of nutrients (glucose, $\mathrm{Na}$ and $\mathrm{K}$ ) in $\mathrm{PN}$, particulate contamination and media-fill tests were checked each day while the machine's settings were only adjusted once a week ( 3 consecutive weeks). Some bottles were changed during the week and other remained in place, according to a predefined protocol. The ACD was installed in a laminar airflow hood GMP Class A with a cleanroom Class $\mathrm{B}$ background and a temperature around $20^{\circ} \mathrm{C}$.

Results Daily operational and performance results:

\begin{tabular}{|c|c|c|c|c|c|c|}
\hline & \multicolumn{2}{|c|}{$0.5 \mathrm{~mL}$} & \multicolumn{2}{|c|}{$40 \mathrm{~mL}$} & \multicolumn{2}{|c|}{$100 \mathrm{~mL}$} \\
\hline & Accuracy & Precision & Accuracy & Precision & Accuracy & Precision \\
\hline Water & $100.9 \%$ & $3.2 \%$ & $98.9 \%$ & $0.3 \%$ & & \\
\hline Nutrient & $99.3-102.7^{*}$ & $2.7-3.9 \%$ * & & & $100-100.4 \%$ * & $0.7-1.5 \%$ * \\
\hline
\end{tabular}

* Depending on nutrient

The concentrations of nutrients in $\mathrm{PN}$ products made weekly always met the specifications (internal limits $\pm 15 \%$ for $\mathrm{Na}, \pm 10 \%$ for glucose and $-15 \%$ to $+10 \%$ for $\mathrm{K})$. No particles or microbiological contamination were detected.

Conclusions Validation proved the acceptable accuracy, precision and aseptic conditions in the course of the week. A sepsis can only be guaranteed by a strict application of GMP in a high quality compounding environment. In those conditions, $\mathrm{PN}$ products can be produced safely for one week with the same settings. Setting it just once a week saves technician time (300 hours/year) and money (15,000 Euro/year).

No conflict of interest.

\section{TCH-052 VALIDATION OF AN AUTOMATED METHOD FOR COMPOUNDING MONOCLONAL ANTIBODY PATIENT DOSES: CASE STUDIES OF BEVACIZUMAB, INFLIXIMAB AND TRASTUZUMAB.}

doi:10.1136/ejhpharm-2013-000276.243

'BMJ Peters, ${ }^{2}$ MAH Capelle, ${ }^{3 \top}$ Arvinte, ${ }^{4} E M W$ van de Garde. 'St. Antonius Hospital, Nieuwegein, The Netherlands; ${ }^{2}$ Therapeomic Inc., Basel, Switzerland; 'University of Geneva, Switzerland; ${ }^{4}$ University of Lausanne, Geneva, Switzerland

Background Automated devices have recently come onto the market as an alternative to manual preparation of drugs for intravenous administration. Automated methods have so far been focused on rapid and time-saving procedures that might harm delicate substances such as monoclonal antibodies (mAbs). Many summaries of product characteristics ( $\mathrm{SmPCs}$ ) of $\mathrm{mAbs}$ state that they require gentle swirling to aid reconstitution and that drawing into a syringe should be done slowly.

Purpose To assess whether automated preparation can be performed with $\mathrm{mAbs}$ without affecting the aggregation state of the proteins.

Materials and Methods Three frequently used mAbs were studied: infliximab (Remicade) and trastuzumab (Herceptin) in lyophilised form, and bevacizumab (Avastin) as a liquid formulation. Brand names are mentioned because biosimilars exist. The effects of different procedures to prepare the patient doses on antibody aggregation were evaluated. Remicade and Herceptin were reconstituted both manually and by an automated arm (i.v.STATION, Health Robotics). Additionally, the effect of vigorous shaking during reconstitution was investigated. The effects of rapid aspiration and dispensing on antibody aggregation were investigated for all three $\mathrm{mAbs}$. The aggregation state was assessed by UV-Vis absorbance, $90^{\circ}$ light scatter, fluorescence spectroscopy, Nile red fluorescence microscopy, and field flow fractionation without cross and focus flow.

Results Samples reconstituted by an automated process showed similar findings compared to manual reconstitution if performed exactly according to the summary of product characteristics (SmPC). Vials that were shaken vigorously showed a significant increase in aggregates. Similarly, rapid aspiration/dispense cycles resulted in a strong increase in the number and sizes of aggregates for all three mAbs; this result was observed after just one rapid aspiration/dispense cycle.

Conclusions Our study showed that automated preparation of $\mathrm{mAbs}$ is feasible if the machine is programmed exactly according to the SmPC, indicating that automated preparation can be used to achieve reproducible high-quality preparation for delicate formulations.

No conflict of interest.

\section{Drug supply/logistics (including: computer-aided drug dispatching and ward pharmacies)}

\section{DSL-001 A MULTIDISCIPLINARY APPROACH TO FURTHER IMPROVEMENTS IN PATIENT SAFETY IN A HOSPITAL WITH COMPUTERISED MEDICAL RECORDS}

doi:10.1136/ejhpharm-2013-000276.244

${ }^{1} \mathrm{C}$ Cuesta-Grueso, ${ }^{1} \mathrm{~J}$ Poquet-Jornet, ${ }^{2} \mathrm{E}$ Flores-Pardo, ${ }^{3} \mathrm{C}$ Santos-Ramírez, ${ }^{4} \mathrm{~A}$ PaciosAlvarez, ${ }^{5}$ MJ Mas-Llull. ${ }^{1}$ Hospital de Denia, Pharmacy, Denia, Spain, ${ }^{2 H o s p i t a l ~ d e ~ D e n i a, ~}$ Quality, Denia, Spain; ${ }^{3}$ Hospital de Denia, Rheumatology, Denia, Spain; ${ }^{4}$ Hospital de Denia, Hematology, Denia, Spain; ${ }^{5}$ Hospital de Denia, nursing supervisor, Denia, Spain

Background Antineoplastic treatments administered at the Medical Day Hospital Unit (MDHU) are high risk for the patient because of their toxicity and mutagenicity and complex pharmacotherapeutic processes. In our hospital medical records are fully computerised and all prescriptions are electronic. So, it is desirable to standardise criteria in a consensus document that minimises variability among professionals to maximise the safety and clinical effectiveness for oncology patients.

Purpose To identify the key points of information that should appear in the consensus document to ensure the correct administration of antineoplastic treatments.

Materials and Methods A multidisciplinary group was created (two physicians, one pharmacist, one nurse and quality mangers). The initial criteria for determining the key points to be imparted were patient safety, clinical effectiveness, organisational coordination and traceability in the Information System. These criteria led to the establishment of 12 key points of information to develop a standard operating procedure (SOP) for each antineoplastic treatment.

Results The 12 key points that were agreed to establish SOPs for each treatment were: indications and usage; prescription form in the Electronic Health Record; pharmaceutical validation to ensure correct indication, dose, volume and type of diluent and infusion time; general and specific nursing indications; contraindications; monitoring of vital signs and anthropometric measures necessary; premedication and time spent on it; preparation of the medicine; possible adverse reactions to the infusion and their management; causes of suspension of treatment; patient information; responsibilities of each professional.

Conclusions The development of SOPs to standardise the pharmacotherapeutic process in the $\mathrm{MDHU}$ contribute to improving the safety and efficiency of antineoplastic treatments. In addition, in a hospital with medical records and where all prescribing is electronic, SOPs contributes to improving the organisation of a complex nursing unit such as the MDHU.

No conflict of interest. 